\title{
Do fall-risk-increasing drugs have an impact on mortality in older hip fracture patients? A population-based cohort study
}

This article was published in the following Dove Press journal:

Clinical Interventions in Aging

29 April 2016

Number of times this article has been viewed

\author{
Annika Kragh Ekstam ${ }^{1,2}$ \\ Sölve Elmståhl' \\ 'Division of Geriatric Medicine, \\ Department of Health Sciences, \\ Lund University, Skåne University \\ Hospital, Malmö, ${ }^{2}$ Department \\ of Medicine, Hässleholm Hospital, \\ Hässleholm, Sweden
}

Objective: The aim of this study was to assess the mortality in hip fracture patients with regard to use of fall-risk-increasing drugs (FRIDs), by comparing survival in exposed and nonexposed individuals.

Design: This was a general population-based cohort study.

Settings: Data on hip fracture patients were retrieved from three national databases.

Participants: All hip fracture patients aged 60 years or older in a Swedish county in 2006 participated in this study.

Measurements: We studied the mortality in hip fracture patients by comparing those exposed to FRIDs, combinations of FRIDs, and polypharmacy to nonexposed patients, adjusting for age and sex. For survival estimates in patients using four or more FRIDs, a Cox regression analysis was used, adjusting for age, sex, and use of any four or more drugs.

Results: First-year all-cause mortality was $24.6 \%(\mathrm{~N}=503)$ in 2,043 hip fracture patients aged 60 years or older, including 170 males (33.8\%) and 333 females (66.2\%). Patients prescribed four or more FRIDs, five or more drugs (polypharmacy), psychotropic drugs, and cardiovascular drugs showed significantly increased first-year mortality. Exposure to four or more FRIDs (518 patients, $25.4 \%$ ) was associated with an increased mortality at 30 days with odds ratios (ORs) 2.01 (95\% confidence interval [CI] 1.44-2.79), 90 days with OR 1.56 (95\% CI 1.19-2.04), 180 days with OR 1.54 (95\% CI 1.20-1.97), and 365 days with OR 1.43 (95\% CI 1.13-1.80). Cox regression analyses adjusted for age, sex, and use of any four or more drugs showed a significantly higher mortality in patients treated with four or more FRIDs at 90 days $(P=0.015)$ and 180 days $(P=0.012)$ compared to patients treated with three or less FRIDs.

Conclusion: First-year all-cause mortality was significantly higher in older hip fracture patients exposed before the fracture to FRIDs, in particular to four or more FRIDs, polypharmacy, psychotropic, and cardiovascular drugs. Interventions aiming to optimize both safety and benefit of drug treatment for older people should include limiting the use of FRIDs.

Keywords: mortality, hip fracture, fall-risk-increasing drugs, population based, older people

\section{Introduction}

Most industrialized countries are facing dramatic demographic changes in the near future, and an increasing aging population will have considerable impact on health care resources. ${ }^{1,2}$ Hip fractures are among the most serious injuries in older persons. Consequences of hip fractures are common with chronic pain, disability, increased need of support in daily activities, and increased morbidity and mortality. ${ }^{3,4}$ Hip fracture patients are in general old, aged more than 80 years, at the time of fracture and are burdened with comorbidity as well as treated with multiple medications. ${ }^{5-7}$ Mortality is
Correspondence: Annika Kragh Ekstam Department of Medicine, Hässleholm Hospital, Esplanadgatan 13, 28I25 Hässleholm, Sweden

Tel +46 44309 I 272

Email annika.kraghekstam@skane.se (c) (5) (5) 2016 Kragh Ekstam and Elmstathl. This work is published and licensed by Dove Medical Press Limited. The full terms of this license are available at https://wwww.dovepress.com/terms.php




high among hip fracture patients, and few interventions have had significant preventive effects on this serious outcome. In comparison with other diagnoses, including cancer and myocardial infarction, mortality is higher among hip fracture patients undergoing fracture surgery. ${ }^{8}$

Risk analyses are routinely carried out in modern health care units to identify older patients at risk of developing malnutrition, falls, pressure ulcers, and infections. Prophylactic interventions are then to be carried out in order to prevent these potentially avoidable complications. ${ }^{9}$ Another prophylactic possibility is prescribing drugs for osteoporosis, which has been shown to have a favorable impact on decreasing both further fractures and mortality. ${ }^{10}$

When caring for older patients, their use of medicines is an important factor causing both complications and adverse effects; however, so far, little has been done to optimize drug treatment in older patients with fractures. An earlier study by the authors on the same group of hip fracture patients showed little evidence of any interventions taken to discontinue or avoid use of fall-risk-increasing drugs (FRIDs). ${ }^{6}$ On the contrary, use of FRIDs increased significantly after the fracture within all drug classes, except for anti-inflammatory drugs that decreased.

It is a difficult task to establish risks vs benefits from drug treatment in the elderly, but fall-risk issues seem too seldom to be in focus when physicians decide on prescribing medications. The challenges that we are facing now due to an increasingly aging population are overwhelming, but to maintain high quality in the care of the elderly, we must set as a first priority preventing the avoidable harm. In the older population, the risks associated with fractures after falls in connection with low-impact trauma and osteoporosis are well known and likely escalate with the use of certain types of drugs. ${ }^{9}$ Medication reviews involving clinical pharmacists are available interventions to identify FRIDs and improve drug prescribing to older people. ${ }^{11,12}$

In this study, we intended to study the associations between older hip fracture patients' medication prior to fracture and first-year mortality aiming to identify the drugs and combinations of drugs that could be potentially unsafe.

\section{Methods}

\section{Study population}

The method of acquiring data for this study has been described in detail in a previous study by the same authors. ${ }^{6}$ This study included all individuals aged 60 years or older diagnosed with a hip fracture and with residency in Skane at the time of fracture. Skane is a county in Sweden with 1.29 million inhabitants and includes $\sim 13 \%$ of the Swedish population.
Patients diagnosed with S72.00, S72.10, S72.11, S72.20, and S72.21, following the International Classification of Diseases, tenth revision, and registered in the administrative database (PASiS) mandatory for Swedish hospitals, from January 1, 2006 to December 31, 2006, were included, and none were excluded. Obtained participant data included date of admission, sex, age, type of fracture, length of in-hospital stay, and time of death during 1 year after admission to hospital. Hip fracture diagnosis, diagnosis of comorbidities, time of death, and prescribed pharmaceuticals were obtained from the Swedish National Board of Health and Welfare's national database of statistics. The unique civic number for all participants was replaced by a case number to anonymize individuals to the investigators.

Data were retrieved from three national databases, namely the Swedish National Patient Register for identifying patients with hip fracture diagnosed within the timeframe, the National Prescription Database for information on drugs prescribed and purchased by the patients during 6 months before the fracture, and the National Cause of Death Register for time of death.

In the National Prescription Database, all prescribed and dispensed drugs must be registered by law, and it covers recipes from physicians in hospitals, outdoor clinics, and general practitioner offices and medications used in most nursing homes. Use of a drug was reported when it had been dispensed once or more during the time limits. Drugs that increase the risk of falls were identified in a previous study from $2011^{6}$ and classified according to the World Health Organization's Anatomical Therapeutic Chemical Classification System into the following drug classes: psychotropic (including sedative/hypnotic, antidepressive, antipsychotic [excluding lithium], and benzodiazepine), cardiovascular (excluding lipid-lowering drugs), anticholinergic, antiepileptic, antiparkinson, and opioids. In addition, combinations of drugs were studied: one, two, three and four or more FRIDs, five or more drugs (polypharmacy), ten or more drugs (major polypharmacy), and the combined use of three or more psychotropic drugs. The analyses also included prophylactic treatment for osteoporosis with oral bisphosphonates and calcium and vitamin D supplements.

The regional research ethics board of Lund University approved this study. Since all data was anonymized by the Swedish National Board of Health and Welfare, patient consent was ensured by advertising in a daily newspaper commonly read within the county. Thus giving participating individuals the possibility to contact the study team for more information. 


\section{Statistical analysis}

All analyses were performed using the Statistical Package for the Social Sciences Version 21.0 and were two sided, with a $P$-value of $<0.05$ regarded as statistically significant. Associations between baseline differences were analyzed using Student's $t$-test or $\chi^{2}$ test. A Cox survival model was used to estimate survival in patients treated with four or more FRIDs compared to those treated with three or less FRIDs. In the regression analyses, adjustment was carried out for age, sex, and use of any kind of four or more drugs (including FRIDs), the categorical variables being age and four or more FRIDs. We analyzed the short-term mortality in the patients, which we set up as 6 months postfracture.

We adjusted for variations in mortality due to differences in age and sex using binary logistic regression. All odds ratios (ORs) for death were adjusted for age and sex. We present $95 \%$ confidence interval (CI) as a measure of precision for the differences in mortality.

\section{Results}

\section{Patients and mortality}

A total of 2,043 hip fracture patients aged 60 years or older admitted to trauma-care hospitals in the Swedish county between January 1, 2006 and December 31, 2006 were included, and none were excluded (Table 1). In 2006, 18,000 hip fractures (all ages) occurred in Sweden, and this study encloses $\sim 11 \%$ of them. Women constituted $73.6 \%$ and men constituted $26.4 \%$ of the population, and the mean age was 83 years. Male patients were younger than female patients, with an average difference of 2.8 years. Significant differences in mortality due to age and sex were present. We found that men had a higher short-term and 1-year mortality compared to women. Data on comorbidities were obtained, but since two large hospitals did not register other diagnoses, except for the hip fracture, these data were not included in the analysis. A total of 503 patients died (24.6\%) within the first year after the hip fracture, 173 patients died $(8.5 \%)$ after 30 days, 304 patients died (14.9\%) after 90 days, and 389 patients died (19.0\%) after 180 days.

\section{Drug exposure and mortality}

Treatment with psychotropic drugs, cardiovascular drugs, and five or more drugs was frequent, and $45.4 \%, 43.8 \%$, and $48.5 \%$ of the patients, respectively, were exposed 6 months prior to the fracture (Table 2).

When studying patients treated with four or more FRIDs, adjusting for age and sex, a statistically significant increase in deaths for those exposed was seen during the first-year postfracture at all measured time intervals. Exposure to four or more FRIDs (518 patients, $25.4 \%$ ) was associated with an increased mortality at 30 days with OR 2.01 (95\% CI 1.442.79), 90 days with OR 1.56 (95\% CI 1.19-2.04), 180 days with OR 1.54 (95\% CI 1.2-1.97), and 365 days with OR 1.43 (95\% CI 1.13-1.80). This was also the case with exposure to five or more drugs ( 990 patients, $48.5 \%$ ) with OR ranging from 1.62 to 1.50 from 30 to 365 days and to cardiovascular drugs (894 patients, $43.8 \%$ ) with OR ranging from 1.67 to 1.43 .

A possible connection between death and drug treatment was also shown in patients exposed to ten or more drugs with OR ranging from 1.72 to 1.62 from 90 to 365 days. For those exposed to any psychotropic drug (928 patients, 45.4\%), mortality increased significantly at 90 days and 365 days with OR 1.30 (95\% CI 1.02-1.67) and OR 1.33 (95\% CI 1.08-1.63), respectively. However, combined treatment with three or more different psychotropic drugs did not show increased mortality in the $242(11.8 \%)$ patients exposed compared to patients not treated with the combination of three or more psychotropic agents.

Table I Characteristics of the study population

\begin{tabular}{|c|c|c|c|c|}
\hline Characteristics & All 2,043, $N$ (\%) & Male 54 I (26.4\%), N (\%) & Female I,502 (73.6\%), N (\%) & $P$-value \\
\hline Age (mean $\pm S D)$ & $83.0 \pm 8.1$ & $81.0 \pm 8.3$ & $83.8 \pm 7.9$ & $<0.0001$ \\
\hline Age $60-79$ years & $576(28)$ & $183(34)$ & $393(26)$ & \\
\hline Age $\geq 80$ years & $\mathrm{I}, 467(72)$ & $358(66)$ & $1,109(74)$ & \\
\hline \multicolumn{5}{|l|}{ Type of fracture ${ }^{a}$} \\
\hline Cervical & I,062 (52) & $272(50)$ & $790(53)$ & 0.498 \\
\hline Pertrochanteric & $839(4 I)$ & $231(43)$ & $608(40)$ & 0.294 \\
\hline Subtrochanteric & $14 \mid(7)$ & $38(7)$ & $103(7)$ & 0.644 \\
\hline \multicolumn{5}{|l|}{ First-year mortality } \\
\hline 30 days & $173(8.5)$ & $62(11.5)$ & III (7.4) & 0.004 \\
\hline 90 days & $304(14.9)$ & $97(17.9)$ & $207(13.8)$ & 0.020 \\
\hline 180 days & $389(19.0)$ & $124(22.9)$ & $265(17.6)$ & 0.070 \\
\hline 365 days & $503(24.6)$ & $170(31.4)$ & $333(22.2)$ & $<0.0001$ \\
\hline Length of stay (mean $\pm S D$ ) & $9.92 \pm 5.75$ & $10.2 \pm 6.3$ & $9.8 \pm 5.5$ & 0.169 \\
\hline
\end{tabular}


Abbreviation: SD, standard deviation. 


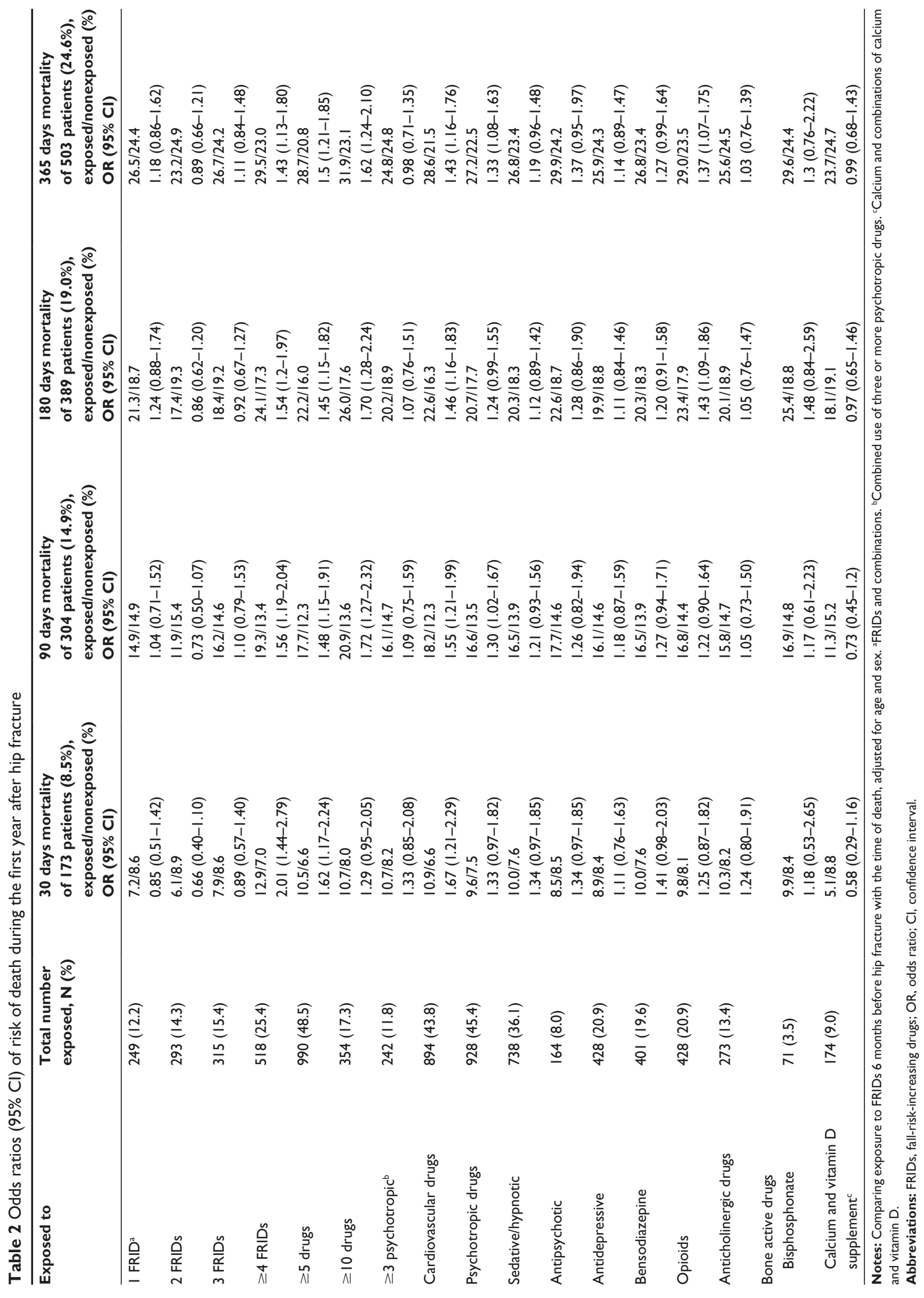


A



B

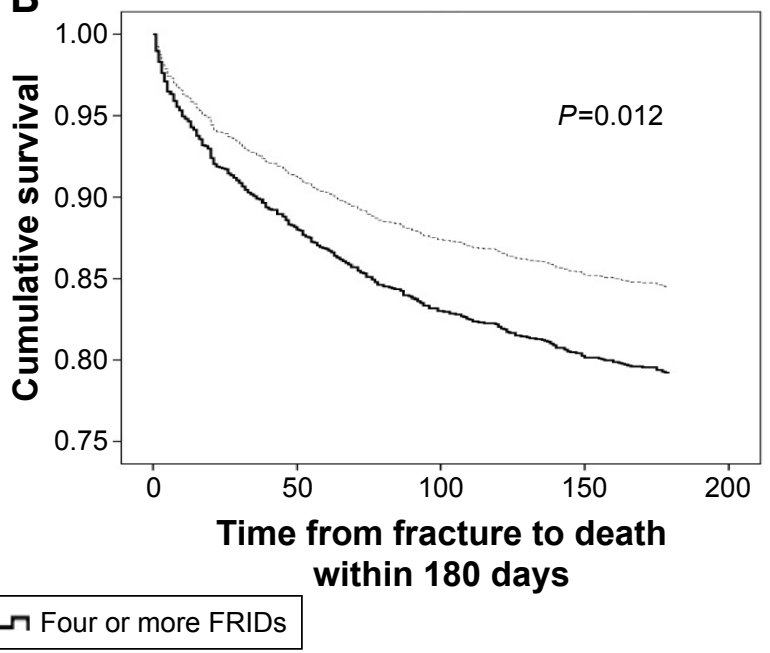

Figure I Time from hip fracture to death within 90 days $(\mathbf{A})$ and 180 days $(\mathbf{B})$ in patients treated with four or more FRIDs compared to patients treated with three or less FRIDs, adjusted for age, sex, and treatment with any four or more drugs.

Abbreviation: FRIDs, fall-risk-increasing drugs.

Significantly increased risk of death during the firstyear postfracture was also seen in patients treated with cardiovascular drugs and opioids. Participants exposed to cardiovascular drugs had significantly increased mortality during 1 year after fracture compared to those not exposed (Table 2), with OR ranging from 1.67 to 1.43 from 30 to 365 days, also adjusting for age and sex.

Treatment with bone-active drugs, such as oral bisphosphonates and calcium with vitamin D supplements, showed no connection to divergent risks of death.

\section{Survival estimates}

Using a Cox regression survival model to the short-term survival, at 90 days and 180 days, when adjusted for age, sex, and treatment with any four or more drugs (including FRIDs), we could show a significant decrease in survival in patients treated with four or more FRIDs, $P=0.015$ ( 90 days) and $P=0.012$ (180 days), compared to patients treated with three or less FRIDs (Figure 1A and B).

\section{Discussion}

In this study, we have found a possible association between increased mortality and use of FRIDs and drug combinations used prior to the fracture event in older hip fracture patients. Patients exposed to medication with four or more FRIDs, polypharmacy (five or more and ten or more drugs), and cardiovascular drugs, and to some extent opioids and psychotropic drugs, showed increased mortality during 1 year after the hip fracture compared to those not exposed. The combination of four or more FRIDs shows up to twofold increased mortality in exposed patients. At 90 days and 180 days postfracture, patients treated with four or more FRIDs had a significantly lower survival rate than patients treated with three or less FRIDs when adjusted for age, sex, and use of four or more of any kind of drugs. When considering drug treatment to older persons, it is important to avoid FRIDs whenever possible, especially combinations of FRIDs.

The advantages of this study are that all older patients suffering from a hip fracture in the general population in one of the larger counties in Sweden are included and none were excluded. The population within the county resides in both urban and rural areas, all emergency hospitals in the region are included, and the diagnostic accuracy on fractures is very precise. Furthermore, data from the national databases cover reliably all fractured patients. In the National Prescription Database, all prescribed and dispensed drugs are registered, and it holds prescriptions from physicians in hospitals, outdoor clinics, and general practitioner offices and most medications used in nursing homes, thus minimizing an underestimation of prescribed drugs. At the time of data collection, 2005-2007, the amount of drugs sold on the Internet was low and nearly nonexistent in the generation involved in this study. However, even if the preciseness of purchases of drugs is high, the knowledge of whether the drugs are consumed or not is lacking. Even so, in our earlier study, we could conclude that the majority of our patients continued getting new recipes and buying the same prescribed drugs 6 months after the fracture, which suggests that the drugs were used. ${ }^{6}$ Since we used prescriptions purchased 6 months 
before the fracture, there is not likely any risk of differential misclassification.

The disadvantages of this study are the lack of information on over-the-counter drugs, comorbidities, and prefracture conditions in the patients. In addition, other factors of importance to mortality in hip fracture patients such as time to surgery, residency before hip fracture, and postoperative complications were not included; however, this was beyond the scope of this register study mainly due to the shortage of available data.

Our results concerning the mortality rates are consistent with other studies on mortality in hip fracture patients both nationally and internationally., ${ }^{4,5,8,13}$ In a study by Kannegaard et $\mathrm{al}^{14}$ on hip fracture patients, they found an excess mortality in men, despite males being younger, and an increased cumulative 1-year mortality compared to the general population in both men and women. As mortality is higher in men and since mean age was higher among women in our study, separate analyses on sex differences were conducted.

Included in FRIDs are medicines used for treating cardiovascular diseases that in themselves are a marker of harmful and potentially lethal conditions. Even so, the higher mortality rate in patients treated with cardiovascular drugs did not exceed the twofold increase shown at 30 days when treating patients with four or more FRIDs, OR 1.67 vs 2.01 within 30 days and OR 1.43 vs 1.43 within 1 year. The connection between excess mortality after hip fracture and cardiovascular disease has been shown in a study by Roche et al. ${ }^{15}$ Our study will not allow for specific interpretation of drug morbidity cause-effect associations. The noted higher mortality associated with cardiovascular drugs could be attributed to the underlying disease but also to the well-known drug related-adverse events, such as hypotension, fatigue, and fall-risk-induced effects associated with mortality. Causes of mortality in older persons are often multifactorial, and organ functions decline with age. This leads to a higher susceptibility to stress such as surgery and hip fracture. Therefore, it is important to study the use of drugs, such as FRIDs, before an event, since besides increasing the risk of fracture; it is also associated with short-term mortality (30-180 days). A case-control study of hip fracture patients noted an increased 9 months' mortality, especially related to cardiovascular death. ${ }^{16}$ Although the increased risk of death after a hip fracture is well studied and documented, the long-term effects of possible causes, such as drug consumption, are not. The intake of FRIDs is indicated by the combination of different types of comorbidities.
The collinearity between drug consumption and morbidity will not enable to disentangle the cause-effect relationship between these components, but this was not the aim of this study and would require a substantially larger study setting. On the other hand, the analysis of a specific drug will not reveal the combined effect of comorbidities and intake of several FRIDs. The strength of this study was the information on drug consumption before the hip fracture occurred, since substantial changes in prescriptions might take place during hospitalization that would confound the analyses.

Besides usage of cardiovascular drugs, treatment with any psychotropic drug also has a negative impact on survival rate. Even though in this study we did not reach full statistical significance, a trend can be perceived in increased mortality in patients using benzodiazepines, which are not often used for treating harmful diseases. ${ }^{17}$ However, we did not observe that a combined treatment with three or more psychotropic drugs in the patients did have any significant association with increased risk of death. Even though this has not been further studied here, one explanation could be that the presence of more severe psychiatric diseases gives the combined treatment more benefits to the patients. As in this study population we were unable to analyze comorbidities, we can only conclude that polypharmacy in itself $(48.5 \%$ treated with five or more drugs) often reflects multimorbidity in hip fracture patients. In a study by Roche et al, ${ }^{15}$ it was shown that the presence of three or more comorbidities was the strongest independent risk factor for increased mortality in older hip fracture patients. With regard to potentially lethal conditions, such as cancer, only $3.2 \%$ of our patients were treated with cytostatic drugs for these conditions. Polypharmacy (five or more drugs) and major polypharmacy (ten or more drugs) have earlier been shown to increase both morbidity and mortality in patients with varying diseases. ${ }^{18-21}$ Here, we found a substantially increased risk of death in patients using five or more drugs (OR 1.62 [95\% CI 1.17-2.24] within 30 days and a $50 \%$ increase in odds of mortality within a year, OR 1.50 [95\% CI 1.21-1.85]). Even though multimedication is a sign of multiple comorbidities and sickness, we could show that combining four or more FRIDs had a significantly higher negative impact on survival in our patients. Earlier studies have shown that it is feasible to reduce drug use in older patients through medication reviews and thus lessen the adverse events due to interactions and adverse reactions. ${ }^{11,12,21}$ When performing medication reviews, we suggest that besides prescribing the lowest effective doses of FRIDs, ending and avoiding treatment with FRIDs should also be highly prioritized. Valuable tools such as Beers criteria, screening 
tool to alert doctors to right treatment, and screening tool of older persons' potentially inappropriate prescriptions, can also be used in improving drug treatment in older people. ${ }^{23,24}$ In addition, the assistance of clinical pharmacists in treating these complex patients should be considered. Even though we could not show it in our study, osteoporosis is a major issue in hip fracture patients, and it is important to identify individuals at risk and treat them according to recommendations. ${ }^{7}$ It is necessary to start a closer collaboration between general practitioners, geriatricians, and orthopedic surgeons in order to maximize the safety and benefit of drug treatment in older hip fracture patients by focusing on FRIDs.

\section{Conclusion}

First-year all-cause mortality was significantly increased in older hip fracture patients exposed to FRIDs before the fracture, especially to four or more FRIDs, polypharmacy, and psychotropic and cardiovascular drugs. To optimize the benefit and safety of drug treatment in older people, attention should be given on limiting the use of FRIDs and treating osteoporosis. Medication reviews are one way of achieving this goal.

\section{Acknowledgments}

The project was supported by independent research grants from Hässleholm Hospital and Skåne County Council's Research and Development Foundation. The grant providers had no role in the design, methods used, data collection, statistical analysis, or article preparation and submission.

\section{Author contributions}

All authors contributed toward data analysis, drafting and critically revising the paper and agree to be accountable for all aspects of the work.

\section{Disclosure}

The authors report no conflicts of interest in this work.

\section{References}

1. Evans JG. Drugs and falls in later life. Lancet. 2003;361:448.

2. Khasraghi FA, Lee EJ, Christmas C, Wenz JF. The economic impact of medical complications in geriatric patients with hip fracture. Orthopedics. 2003;26:49-53. [discussion 53].

3. van Balen R, Steyerberg EW, Polder JJ, Ribbers TL, Habbema JD, Cools HJ. Hip fracture in elderly patients: outcomes for function, quality of life, and type of residence. Clin Orthop Relat Res. 2001:232-243.

4. Vestergaard P, Rejnmark L, Mosekilde L. Increased mortality in patients with a hip fracture-effect of pre-morbid conditions and post-fracture complications. Osteoporos Int. 2007;18:1583-1593.

5. Kanis JA, Oden A, Johnell O, De Laet C, Jonsson B, Oglesby AK. The components of excess mortality after hip fracture. Bone. 2003;32: $468-473$.
6. Kragh A, Elmstahl S, Atroshi I. Older adults' medication use 6 months before and after hip fracture: a population-based cohort study. J Am Geriatr Soc. 2011;59:863-868.

7. Magaziner J, Lydick E, Hawkes W, et al. Excess mortality attributable to hip fracture in white women aged 70 years and older. Am J Public Health. 1997;87:1630-1636.

8. Johnell O, Kanis JA, Oden A, et al. Mortality after osteoporotic fractures. Osteoporos Int. 2004;15:38-42.

9. Bennett A, Gnjidic D, Gillett M, et al. Prevalence and impact of fallrisk-increasing drugs, polypharmacy, and drug-drug interactions in robust versus frail hospitalised falls patients: a prospective cohort study. Drugs Aging. 2014;31:225-232.

10. Tang BM, Eslick GD, Nowson C, Smith C, Bensoussan A. Use of calcium or calcium in combination with vitamin $\mathrm{D}$ supplementation to prevent fractures and bone loss in people aged 50 years and older: a meta-analysis. Lancet. 2007;370:657-666.

11. Bondesson A, Eriksson T, Kragh A, Holmdahl L, Midlöv P, Höglund P. In-hospital medication reviews reduce unidentified drug-related problems. Eur J Clin Pharmacol. 2013;69:647-655.

12. van der Velde N, Stricker BH, Pols HA, van der Cammen TJ. Risk of falls after withdrawal of fall-risk-increasing drugs: a prospective cohort study. Br J Clin Pharmacol. 2007;63:232-237.

13. Abrahamsen B, van Staa T, Ariely R, Olson M, Cooper C. Excess mortality following hip fracture: a systematic epidemiological review. Osteoporos Int. 2009;20:1633-1650.

14. Kannegaard PN, van der Mark S, Eiken P, Abrahamsen B. Excess mortality in men compared with women following a hip fracture. National analysis of comedications, comorbidity and survival. Age Ageing. 2010;39:203-209.

15. Roche JJ, Wenn RT, Sahota O, Moran CG. Effect of comorbidities and postoperative complications on mortality after hip fracture in elderly people: prospective observational cohort study. BMJ. 2005; 331:1374.

16. Cameron ID, Chen JS, March LM, et al. Hip fracture causes excess mortality owing to cardiovascular and infectious disease in institutionalized older people: a prospective 5-year study. J Bone Miner Res. 2010; 25:866-872.

17. Gosch M, Wortz M, Nicholas JA, Doshi HK, Kammerlander C, Lechleitner M. Inappropriate prescribing as a predictor for long-term mortality after hip fracture. Gerontology. 2014;60:114-122.

18. Hajjar ER, Cafiero AC, Hanlon JT. Polypharmacy in elderly patients. Am J Geriatr Pharmacother. 2007;5:345-351.

19. Maher RL, Hanlon J, Hajjar ER. Clinical consequences of polypharmacy in elderly. Expert Opin Drug Saf. 2014;13:57-65.

20. Nobili A, Licata G, Salerno F, et al; SIMI Investigators. Polypharmacy, length of hospital stay, and in-hospital mortality among elderly patients in internal medicine wards. The REPOSI Study. Eur JClin Pharmacol. 2011; 67:507-519.

21. Strand LM, Morley PC, Cipolle RJ, Ramsey R, Lamsam GD. Drug-related problems: their structure and function. DICP. 1990;24:1093-1097.

22. van der Velde N, van den Meiracker AH, Pols HA, Stricker BH, van der Cammen TJ. Withdrawal of fall-risk-increasing drugs in older persons: effect on tilt-table test outcomes. J Am Geriatr Soc. 2007;55: 734-739.

23. Panel American Geriatrics Society Beers Criteria Update Expert. American geriatrics society updated beers criteria for potentially inappropriate medication use in older adults. J Am Geriatr Soc. 2012;60: 616-631.

24. Hill-Taylor B, Sketris I, Hayden J, Byrne S, O'Sullivan D, Christie R. Application of the STOPP/START criteria: a systematic review of the prevalence of potentially inappropriate prescribing in older adults, and evidence of clinical, humanistic and economic impact. J Clin Pharm Ther. 2013;38:360-372. 


\section{Publish your work in this journal}

Clinical Interventions in Aging is an international, peer-reviewed journal focusing on evidence-based reports on the value or lack thereof of treatments intended to prevent or delay the onset of maladaptive correlates of aging in human beings. This journal is indexed on PubMed Central, MedLine,

CAS, Scopus and the Elsevier Bibliographic databases. The manuscript management system is completely online and includes a very quick and fair peer-review system, which is all easy to use. Visit http://www.dovepress. com/testimonials.php to read real quotes from published authors. 